\title{
Arthroscopic os trigonum en-bloc removal outcomes at 6-month follow-up are better compared to Stieda's process resection in patients with posterior ankle impingement syndrome: a prospective comparative study
}

\author{
Panagiotis D Symeonidis, ${ }^{1}$ Emmanouil T Papakostas, ${ }^{2,3}$ \\ Theodorakys Marín Fermín (1) , ${ }^{3,4}$ Maria Tsatlidou, ${ }^{1}$ Ioannis Terzidis, ${ }^{1,3}$ \\ Pericles Papadopoulos ${ }^{5}$
}

\begin{abstract}
'St. Luke's Hospital, Thessaloniki, Greece

${ }^{2}$ Aspetar Orthopaedic and Sports Medicine Hospital, Doha, Qatar

${ }^{3}$ Sports Medicine Orthopaedics, TheMIS Orthopaedic Center,

Thessaloniki, Greece

${ }^{4}$ Department of Traumatology, Hospital Universitario Periférico de Coche, Caracas, Venezuela ${ }^{5}$ 2nd Orthopedic Department, General Hospital of Thessaloniki Gennimatas, Thessaloniki, Greece
\end{abstract}

Correspondence to Dr Theodorakys Marín Fermín, Department of Traumatology, Hospital Universitario Periférico de Coche, Caracas, Venezuela; theodorakysmarin@yahoo.com

Accepted 19 May 2021 Published Online First 30 June 2021

Check for updates

(C) International Society of Arthroscopy, Knee Surgery and Orthopaedic Sports Medicine 2021. No commercial re-use. See rights and permissions. Published by BMJ.

To cite: Symeonidis PD,

Papakostas ET, Marín

Fermín T, et al. J ISAKOS

2021:6:329-332.

\section{ABSTRACT}

Objective To evaluate the potential differences in American Orthopaedic Foot and Ankle Society (AOFAS) hindfoot score and Foot Function Index (FFI) at 6-month and 12-month postoperative follow-up of arthroscopic treatment for posterior ankle impingement (PAIS) between os trigonum (OT) and Stieda's process (SP) patients

Methods Thirty consecutive patients (32 ankles) treated in our Institution for PAIS with posterior arthroscopy were prospectively enrolled in the study from December 2012 to July 2019. Indications were patients with PAIS with persistent symptoms following conservative management. Exclusion criteria were the coexistence of concomitant pathologies and patients who underwent additional surgical procedures. An independent investigator interviewed and evaluated the patients according to the AOFAS hindfoot score and FFI preoperatively, at 6-month and 12-month follow-up. Results Except for AOFAS scores in the SP group (MD (mean difference) 11.28, $p=0.08$ ), patients undergoing arthroscopic treatment for bony PAIS had an overall significant improvement in AOFAS score (OT MD 22.29, $p<0.05)$ and FFI (OT MD -70.07, $p<0.05$; SP MD $-50.96, p<0.05)$ from their preoperative scores at 6-month follow-up. Similarly, a significant improvement in AOFAS score (OT MD 5.78, $p=0.01$; SP MD 12.14, $p<0.05)$ and FFI (OT MD -9.36, $p=0.04$; SP MD -26.43, $p<0.05$ ) was observed from the 6 -month to 12 -month follow-up in all groups. At 6-month follow-up, the OT group had significantly better FFI outcomes (MD -33.57, $p=0.04$ ) compared with the SP group. No differences were found by group when comparing AOFAS score and FFI score at 12-month follow-up.

Conclusions When comparing patients undergoing OT excision or SP resection, better FFI outcomes were observed in the OT group at 6-month follow-up. Level of evidence Prospective comparative study. Level II.

\section{INTRODUCTION}

Posterior ankle impingement syndrome (PAIS) is characterised by posterior ankle pain during forced plantar flexion. ${ }^{1}$ It is a prevalent disorder in the athletic population from repeated plantar flexion. ${ }^{2-4}$ It can also result from acute trauma in fractures in the general population. ${ }^{5-7}$ Osseous impingement is

\section{What are the new findings}

- Patients undergoing arthroscopic treatment for bony posterior ankle impingement syndrome had an overall significant American Orthopaedic Foot and Ankle Society (AOFAS) score and Foot Function Index (FFI) improvement at 6-month follow-up.

- When comparing the os trigonum versus Stieda's process group at 6-month follow-up, the os trigonum group had significantly better AOFAS and FFI outcomes.

- No differences were found by group when comparing AOFAS score and FFI score at 12-month follow-up.

the more frequent type, where either an os trigonum (OT) and or a hypertrophic posterior talar process, the so-called Stieda's process (SP) result in irritation of the surrounding soft tissues during vigorous activities. ${ }^{189}$ Among the affected anatomical structures, the flexor hallucis longus (FHL) is involved in more than two-thirds of the cases. ${ }^{10-12}$

The origin of anatomical variations, such as the OT and SP, is at the posterior talar nucleus ossification phase between 8 and 11 years of age. ${ }^{13} 14$ Histologically, OT represents an accessory ossicle partially united through a fibrous or cartilaginous bridge to the posterior talus and is present in $5 \%-15 \%$ of normal feet. ${ }^{1914}$ On the other hand, SP results from its complete fusion to the talus and represents a hypertrophic posterior talar process. ${ }^{15}$ Both variants are usually asymptomatic. ${ }^{2} 10 \quad 13$ Similar to other impingement syndromes, surgical treatment of PAIS is advocated after the failure of conservative management. ${ }^{911} 14$ Several approaches have been proposed, with a growing interest in minimally invasive techniques. ${ }^{13}$ Posterior ankle arthroscopy is reported to have lower complication rates and a faster return to full activities. ${ }^{8} 14$

Since the introduction and popularisation of posterior ankle arthroscopy by van Dijk et al, ${ }^{16}$ several studies have reported its implementation in PAIS treatment. ${ }^{239}$ However, it remains unclear if there is a difference in outcome between the OT and SP bony impingement variants. The objective of the present study is to evaluate the potential 
differences in American Orthopaedic Foot and Ankle Society (AOFAS) hindfoot score and Foot Function Index (FFI) at 6-month and 12-month postoperative follow-up of arthroscopic treatment for PAIS between OT and SP patients. Our hypothesis was that the en-bloc arthroscopic removal of OT would have better functional outcomes than SP resection.

\section{METHODS}

Following Institutional Board Ethics approval, 30 consecutive patients (32 ankles) who were treated in our institution for PAIS with posterior arthroscopy were prospectively enrolled in the study over 7 years (December 2012 to July 2019). Two orthopaedic surgeons performed all procedures, a foot and ankle specialist (PDS) and a sports traumatologist specialised in arthroscopic surgery (ETP). Both surgeons followed the same operative technique and agreed on the same postoperative protocol before the study initiation. Indications for the procedure were patients with a clinical diagnosis and MRI and/or CT findings of PAIS with persistent symptoms following a minimum of 3 months of conservative management. This included physiotherapy, modification of shoes and activities and single ultrasound-guided betamethasone injections in selected patients.

Exclusion criteria were the coexistence of concomitant pathologies in the region, such as osteochondral lesions of the talar dome, and patients who underwent additional surgical procedures in the same setting. Among others, these included ankle ligament repairs and Achilles or peroneal tendons procedures. Patients with an acute posterior process fracture (the so-called Shepherd's fracture) were also excluded from the study. On the other hand, FHL pathology presence such as stenosing tenosynovitis and/or a low riding muscle belly was not considered an exclusion criterion. Baseline demographic data and preoperative AOFAS hindfoot score and FFI were recorded (table 1).

\section{Operative technique}

Patients who met the inclusion criteria underwent a standard posterior ankle arthroscopy as described by van Dijk et al. ${ }^{16}$ Diagnostic of OT or SP was done under arthroscopic visualisation, confirming the preoperative diagnosis in all cases. All operations were performed under thigh tourniquet and regular intravenous antibiotics in the prone position. En-bloc removal was achieved in all OT patients and 8 out of the 14 SP patients. A combination of shavers, burrs, arthroscopic scissors and osteotomes was used in each case. At the end of each procedure, the FHL tendon was visible and unobstructed in its excursion. Postoperatively, patients were discharged with the operated ankle in a soft dressing. They were mobilised on crutches in a partial weight-bearing status for the first week with range-ofmotion exercises, routine cryotherapy and oral analgesics. By the end of the second week postoperatively, they were allowed to full weight-bearing. Further rehabilitation was individualised according to each patient's recovery and activity level. All relevant short-term and long-term complications previously reported in the literature were screened and recorded during the first postoperative year.

\section{Outcome measures}

An independent investigator, not involved in any operations, interviewed and evaluated the patients according to the AOFAS hindfoot score and FFI preoperatively and at 6-month and 12-month follow-up. The AOFAS hindfoot score is an outcome measure that evaluates patients' pain, function and alignment based on clinicians' observations. Although it is not validated, its widespread use in the past two decades makes it a valuable tool for meta-analyses and comparative studies alike. The FFI is a validated, self-administered index that measures foot pathology's impact on function in pain, disability and activity restriction. ${ }^{17}$

\section{Statistical analysis}

Levene's test was implemented to test for homogeneity of variance of sex, age and preoperative AFOFAS hindfoot score and FFI. A two-tailed paired t-test was used to evaluate improvement in AOFAS score and FFI from preoperative to 6-month and 12-month follow-up by group. A two-tailed independent t-test was used to compare preoperative, postoperative AOFAS score and FFI at 6-month and 12-month follow-up between groups. For patients with bilateral involvement, each ankle was considered as independent patients for statistical purposes. $\mathrm{P}<0.05$ were considered statistically significant. SPSS V.19 was used to perform statistical analysis. Results were presented as mean with $\mathrm{SD}$, ranges, mean differences $(\mathrm{MD})$ and $95 \%$ confidence intervals $(\mathrm{CI})$

\section{RESULTS}

Twenty-six patients (28 ankles) were included in the final analysis. There were two patients with bilateral involvement that were operated on at a different stage. There were 16 male and 10 female patients. Their mean age at the time of surgery was $28.96 \pm 9.47$ years (range 15-48). Out of the initial 30 patients, three patients developed concomitant pathologies in the region during the study period and were excluded from further evaluation. In detail, these patients were respectively a case of lateral ankle instability, a Haglund deformity and an osteochondral lesion of the talus. One patient was lost in follow-up.

Postoperative outcomes at 6-month and 12-month follow-up were summarised (tables 1 and 2). Surgical groups had no significant differences in terms of age, sex, preoperative AOFAS

\begin{tabular}{|c|c|c|c|c|c|c|c|c|}
\hline \multirow[b]{2}{*}{ Group } & \multirow[b]{2}{*}{ Age (years) } & \multirow[b]{2}{*}{ Sex } & \multicolumn{2}{|c|}{ Preoperative scores } & \multicolumn{2}{|c|}{ 6-month follow-up outcomes } & \multicolumn{2}{|c|}{ 12-month follow-up outcomes } \\
\hline & & & AOFAS & FFI & AOFAS & FFI & AOFAS & FFI \\
\hline Os trigonum & $\begin{array}{l}26.46 \pm 7.25 \\
(15-41)\end{array}$ & $\begin{array}{l}6 \text { males } \\
7 \text { females }\end{array}$ & $\begin{array}{l}64.50 \pm 14.70 \\
(25-83)\end{array}$ & $\begin{array}{l}96.00 \pm 45.51 \\
(30-193)\end{array}$ & $\begin{array}{l}86.79 \pm 11.17 \\
(63-100)\end{array}$ & $\begin{array}{l}25.93 \pm 30.81 \\
(0-100)\end{array}$ & $\begin{array}{l}92.57 \pm 10.09 \\
(72-100)\end{array}$ & $\begin{array}{l}16.57 \pm 27.92 \\
(0-99)\end{array}$ \\
\hline Stieda's process & $\begin{array}{l}31.46 \pm 10.70 \\
(17-48)\end{array}$ & $\begin{array}{l}10 \text { males } \\
3 \text { females }\end{array}$ & $\begin{array}{l}63.79 \pm 18.56 \\
(29-91)\end{array}$ & $\begin{array}{l}110.46 \pm 54.46 \\
(35-243)\end{array}$ & $\begin{array}{l}75.07 \pm 17.48 \\
(33-100)\end{array}$ & $\begin{array}{l}59.50 \pm 46.27 \\
(2-189)\end{array}$ & $\begin{array}{l}87.21 \pm 17.68 \\
(33-100)\end{array}$ & $\begin{array}{l}33.07 \pm 46.22 \\
(0-189)\end{array}$ \\
\hline$P$ value & 0.07 & 0.10 & 0.25 & 0.55 & 0.052 & $0.04 \dagger$ & 0.35 & 0.28 \\
\hline
\end{tabular}

*Data are expressed as mean \pm SD with range in parentheses unless otherwise indicated. 
Table 2 Preoperative scores, and postoperative outcomes at 6-month and 12-month follow-up* of arthroscopic os trigonum removal, Stieda's process resection and combined group in posterior ankle impingement syndrome

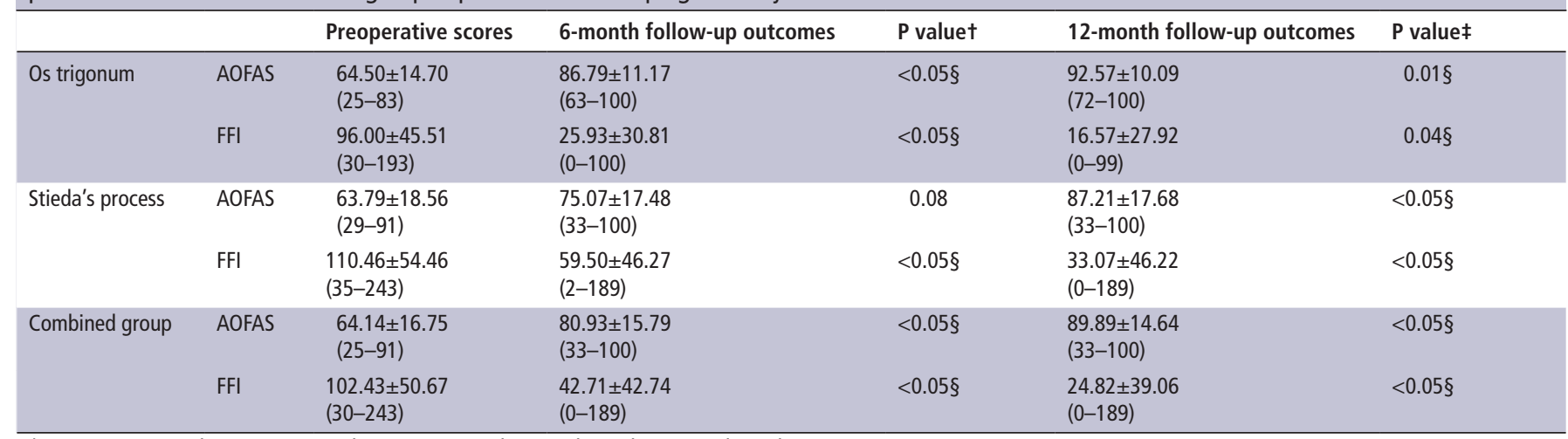

${ }^{*}$ Data are expressed as mean \pm SD with range in parentheses unless otherwise indicated.

tPreoperative scores vs 6-month follow-up outcomes.

\#6-month vs 12 -month follow-up outcomes.

$\S$ Statistical significance.

AOFAS, The American Orthopedic Foot and Ankle Society Score; FFI, Foot Function Index.

score and FFI. With the exception of AOFAS scores in the SP group (MD 11.28, 95\% CI 64.98 to 85.16), patients undergoing arthroscopic treatment for bony PAIS had an overall significant improvement in AOFAS score (OT MD 22.29, 95\% CI 80.34 to 93.24 ) and FFI (OT MD $-70.07,95 \% \mathrm{CI}$ 8.14 to 43.72 ; SP MD $-49.36,95 \%$ CI 32.78 to 86.22 ) from their preoperative scores at 6 -month follow-up, irrespective of the subgroup. Similarly, a significant improvement in AOFAS score (OT MD 5.78, 95\% CI 86.74 to 98.40; SP MD 12.14, $95 \%$ CI 77.00 to 97.42 ) and FFI (OT MD -9.36, 95\% CI 0.45 to 32.69 ; SP MD $-26.43,95 \%$ CI 6.38 to 59.76 ) was observed from the 6-month to 12-month follow-up in all groups.

At a 6-month follow-up, the OT group had significantly better FFI outcomes (MD -33.57, 95\% CI 8.14 to 43.72) compared with the SP group. Similarly, OT group had better AOFAS score at 6-month follow-up (MD 11.72, 95\% CI 80.34 to 93.24), and AOFAS score (MD 5.36, 86.74 to 98.40) and FFI (MD -16.5, 0.45 to 32.69 ) at 12 -month follow-up, but the differences were not statistically significant. There were no major complications in this study population.

\section{DISCUSSION}

The current prospective cohort study's main finding is that patients with PAIS undergoing OT excision or SP resection had similar AOFAS and FFI outcomes at 12-month follow-up. However, better FFI outcomes (MD -33.57, 95\% CI 8.14 to 43.72) were observed at 6-month follow-up in the OT group. Except for the AOFAS scores (MD 11.28, 95\% CI 64.98 to 85.16 ) in the SP group, patients undergoing arthroscopic treatment for bony PAIS had a significant improvement at 6 months $(\mathrm{p}<0.05)$. This improvement continues to be significant from 6 -month to 12-month follow-up in all patients $(\mathrm{p}<0.05)$.

A plausible explanation of these findings is the nature of each procedure. Inevitably, the resection of an SP results in an area of exposed bleeding cancellous bone in the posterior aspect of the talus. This may lead to more posterior ankle swelling and discomfort, which can influence the initial postoperative rehabilitation.

Surgical treatment of PAIS has been extensively reported in the literature with good to excellent outcomes in diverse approaches. $^{24-691013151819}$ In a systematic review, Spennacchio et $a l^{8}$ reported clinical improvement after operative treatment in more than 250 OT excisions (15 studies), with a mean postoperative increase of 33.69 points in the AOFAS score. Numerous recently published case series support these findings. ${ }^{2} 67111419$ Dinato et $\mathrm{al}^{7}$ revealed an AOFAS score improvement of 27.8 points in 32 amateur and professional athletes after a minimum 2-year follow-up, with a mean time to return to sports of $15.6 \pm 13.7$ and $16.3 \pm 9$ weeks, respectively. Likewise, Heyer et $a l,{ }^{2}$ in a retrospective case series of 38 dancers, demonstrated a $90.8 \%$ pain improvement after open posteromedial OT excision and FHL tenolysis, and a $94.6 \%$ return to a preoperative level of dance. Morelli et al ${ }^{14}$ reported an increase in both Tegner scale score ( 4.7 points) and AOFAS score (28.2 points) at a mean follow-up of 38.9 months in professional dancers. Last, Carreira et $a l^{6}$ reported a significant improvement in the Visual Analogue Scale (MD - 4.5 points) and AOFAS score (MD 19.9 points) at 1-year follow-up $(\mathrm{p}<0.01)$ in 19 competitive athletes, but no differences in Tegner scale score.

The advantages of minimally invasive techniques in the treatment of PAIS have been highlighted in the literature, ${ }^{4} 101420$ as arthroscopic approaches tend to have lower complication rates ( $<7 \%$ for major and $<2 \%$ for minor complications) and a faster return to full activities (6 weeks). ${ }^{8}$ Previous studies have reported an improvement in AOFAS scores from baseline scores after arthroscopic/endoscopic treatment of bony PAIS. ${ }^{9} 1019-21$ The prospective cohort study of Scholten et $a l^{21}$ revealed an MD of 18.5 points at a mean follow-up of 36 months. Likewise, Weiss et $a l^{10}$ reported a MD of 33 points at a mean follow-up of 26 months, and in Cuéllar-Avaroma et $a l^{19}$ series, the MD was found 20.49 points at a mean follow-up of 27.1 months. Lastly, Georgiannos et al, in a randomised controlled trial, revealed a MD of 26.57 points at a 5-year follow-up. Our results are in accordance with such findings, as a significant improvement of AOFAS (MD 25.75 points, 95\% CI 84.21 to 95.57) and FFI (MD -77.61 points, $95 \%$ CI 9.67 to 39.97 ) was observed during the first year after arthroscopic surgery in the current series.

Comparative studies regarding different aetiologies of PAIS are scarce. $^{2122}$ The study by Calder et $a l^{22}$ contrasted outcomes between soft tissue and bony impingement. Return to training was significantly faster in the soft tissue impingement group, but no differences were found in the return-to-sports rates. On the other hand, Scholten et al ${ }^{21}$ compared posterior arthroscopy outcomes between overuse and acute post-traumatic injuries. They observed a faster return to sports rates (8 weeks), higher satisfaction $(84 \%$ excellent or good) and AOFAS scores (median 100, $\mathrm{p}=0.03$ ) in 
the overuse group. However, no differences were found when comparing soft tissue and bony impingement groups.

Theoretically, en-bloc removal of OT can be performed with more ease compared with SP resection. In any case, boundaries of bony landmarks should be defined before resection ${ }^{23}$ to avoid inadvertent injury to the posterior talar dome cartilage, bone, posterior talofibular ligament and surrounding soft tissues. ${ }^{3} 41024$ This is particularly important when multiple attempts are made in a piece-by-piece resection. ${ }^{10}$ Bony impingement resection with the ankle in dorsiflexion has been advocated, as it exposes the posterior talar dome, and boundaries for resection can be carefully chosen. ${ }^{3}$ We have also found this manoeuvre convenient, especially in patients with a large SP removed in a piecemeal manner.

\section{Limitations}

This study had some limitations. First, it was a non-randomised study with a relatively small number of patients. Furthermore, the OT and SP sizes were not recorded in each case. This parameter could have influenced the clinical outcome. Finally, an FHL pathology, when present, was neither quantified nor was it evaluated separately.

\section{CONCLUSION}

Except for the SP group's AOFAS score, arthroscopic treatment of bony PAIS provided a significant AOFAS score and FFI improvement at a 6-month follow-up. Additionally, a significant AOFAS score and FFI improvement were observed from 6-month to 12-month follow-up. Finally, when comparing patients undergoing OT excision or SP resection, better FFI outcomes were observed in the OT group at a 6-month follow-up. No differences were found by groups at 12-month follow-up.

Correction notice Since Online First publication, author name 'Maria Tsalidou' has been corrected to 'Maria Tsatlidou'.

Twitter Theodorakys Marín Fermín @anatheomia

Contributors PDS: conceptualisation, methodology, investigation, writing-review and editing, supervision, project administration. ETP: investigation, writing - review and editing, supervision. TMF: formal analysis, writing —original draft, visualisation. MT: investigation. IT and PP: writing — review and editing, supervision.

Funding The authors have not declared a specific grant for this research from any funding agency in the public, commercial or not-for-profit sectors.

Competing interests None declared.

Patient consent for publication Not required.

Provenance and peer review Not commissioned; externally peer reviewed.

Data availability statement All data relevant to the study are included in the article or uploaded as supplementary information. All data will be available on reasonable request.

ORCID iD

Theodorakys Marín Fermín http://orcid.org/0000-0002-1698-9517

\section{REFERENCES}

1 Miyamoto W, Takao M, Matsushita T. Hindfoot endoscopy for posterior ankle impingement syndrome and flexor hallucis longus tendon disorders. Foot Ankle Clin 2015;20:139-47

2 Heyer JH, Rose DJ. Os Trigonum excision in dancers via an open posteromedial approach. Foot Ankle Int 2017;38:27-35.

3 Vilá J, Vega J, Mellado M, et al. Hindfoot endoscopy for the treatment of posterior ankle impingement syndrome: a safe and reproducible technique. Foot Ankle Surg 2014;20:174-9.

4 Smyth NA, Murawski CD, Levine DS, et al. Hindfoot arthroscopic surgery for posterior ankle impingement: a systematic surgical approach and case series. Am I Sports Med 2013;41:1869-76.

5 Guo QW, Hu YL, Jiao C, et al. Open versus endoscopic excision of a symptomatic OS trigonum: a comparative study of 41 cases. Arthroscopy 2010;26:384-90.

6 Carreira DS, Vora AM, Hearne KL, et al. Outcome of arthroscopic treatment of posterior impingement of the ankle. Foot Ankle Int 2016;37:394-400.

7 Dinato MCME, Luques IU, Freitas MdeF, et al. Endoscopic treatment of the posterior ankle impingement syndrome on amateur and professional athletes. Knee Surg Sports Traumatol Arthrosc 2016;24:1396-401.

8 Spennacchio P, Cucchi D, Randelli PS, et al. Evidence-based indications for hindfoot endoscopy. Knee Surg Sports Traumatol Arthrosc 2016;24:1386-95.

9 Georgiannos D, Bisbinas I. Endoscopic versus open excision of os Trigonum for the treatment of posterior ankle impingement syndrome in an athletic population: a randomized controlled study with 5-year follow-up. Am I Sports Med 2017:45:1388-94.

10 Weiss WM, Sanders EJ, Crates JM, et al. Arthroscopic excision of a symptomatic OS Trigonum. Arthroscopy 2015;31:2082-8.

11 Kudaş S, Dönmez G, Ișık Çetin, et al. Posterior ankle impingement syndrome in football players: case series of 26 elite athletes. Acta Orthop Traumatol Turc 2016:50:649-54

12 Sharpe BD, Steginsky BD, Suhling $M$, et al. Posterior ankle impingement and flexor Hallucis longus pathology. Clin Sports Med 2020;39:911-30.

13 Galla M, Lobenhoffer P. Technique and results of arthroscopic treatment of posterior ankle impingement. Foot Ankle Surg 2011;17:79-84.

14 Morelli F, Mazza D, Serlorenzi P, et al. Endoscopic excision of symptomatic OS Trigonum in professional dancers. J Foot Ankle Surg 2017;56:22-5.

15 Abramowitz Y, Wollstein R, Barzilay Y, et al. Outcome of resection of a symptomatic OS trigonum. J Bone Joint Surg Am 2003;85:1051-7.

16 van Dijk CN, Scholten PE, Krips R. A 2-portal endoscopic approach for diagnosis and treatment of posterior ankle pathology. Arthroscopy 2000;16:871-6.

17 Martin RL, Irrgang JJ, Lalonde KA, et al. Current concepts review: foot and ankle outcome instruments. Foot Ankle Int 2006;27:383-90.

18 Ogut T, Ayhan E, Irgit K, et al. Endoscopic treatment of posterior ankle pain. Knee Surg Sports Traumatol Arthrosc 2011;19:1355-61.

19 Cuéllar-Avaroma A, King-Hayata MA, Martínez-de Anda MC, et al. [Endoscopic treatment for the posterior impingement of the ankle]. Acta Ortop Mex 2017;31:24-9

20 Ahn JH, Kim Y-C, Kim H-Y. Arthroscopic versus posterior endoscopic excision of a symptomatic OS trigonum: a retrospective cohort study. Am I Sports Med 2013;41:1082-9.

21 Scholten PE, Sierevelt IN, van Dijk CN. Hindfoot endoscopy for posterior ankle impingement. J Bone Joint Surg Am 2008;90:2665-72.

22 Calder JD, Sexton SA, Pearce CJ. Return to training and playing after posterior ankle arthroscopy for posterior impingement in elite professional soccer. Am I Sports Med 2010;38:120-4.

23 Lui TH. Arthroscopic treatment of posterior ankle impingement in the supine position using coaxial posterior portals. Foot Ankle Int 2014;35:834-7.

24 van Dijk CN, de Leeuw PAJ, Scholten PE. Hindfoot endoscopy for posterior ankle impingement. surgical technique. J Bone Joint Surg Am 2009;91 Suppl 2:287-98. 